\title{
Web Applications for Interoperability of Biodiversity Data in France
}

\author{
Julien Corny ${ }^{\ddagger}$, El-Makki Voundy ${ }^{\ddagger}$, Camille Monchicourt ${ }^{\S}$, Amandine Sahll, Olivier Rovellotti ${ }^{\ddagger}$ \\ ‡ Natural Solutions, Marseille, France \\ $\S$ Parc national des Ecrins, Gap, France \\ | Parc national des Cévennes, Florac, France
}

Corresponding author: Julien Corny (julien corny@natural-solutions.eu)

Received: 04 Jul 2019 | Published: 10 Jul 2019

Citation: Corny J, Voundy E-M, Monchicourt C, Sahl A, Rovellotti O (2019) Web Applications for Interoperability of Biodiversity Data in France. Biodiversity Information Science and Standards 3: e37947.

https://doi.org/10.3897/biss.3.37947

\begin{abstract}
In the context of the French law for the reconquest of biodiversity (Legifrance 2016), public and private stakeholders must share environmental impact assessment data as open data to the French National Inventory of the Natural Heritage (Muséum national d'Histoire naturelle 2019). In order to achieve this, the Information System for Nature and Landscape (SINP) provided standards and guidelines for protocols, taxonomy, and metadata in order to comply with the FAIR (Findability, Accessibility, Interoperability, Reusability; Wilkinson et al. 2016) concept of data management. However, private institutions, who must run environmental impact assessments, can be confused by the number of technical details and the high level of data literacy needed to comply with these standards. Here, we will present several tools (GeoNature 2019, Natural Solutions 2019) that we are currently developing to facilitate the raw biodiversity data conversion and export using SINP standards (Jomier et al. 2018). Although SINP and Darwin Core (Wieczorek et al. 2012) standards share common concepts and properties, SINP standards focus on data reusability in the framework of French environmental programs, resulting in the creation of specific mandatory attributes (Chataigner et al. 2014). Our tools perform extract, transform and load (ETL) operations as well as RDF (Resource Description Framework) exports using ad-hoc ontology adapted to the specificities of the SINP standard. Finally, we observed that despite the success of the process (after one year, nearly one thousand
\end{abstract}


datasets are available on the SINP web platform), several issues still need to be addressed, including data quality issues, which could hamper data reuse by stakeholders.

\section{Keywords}

open-data, web, standard, ETL, data management

\section{Presenting author}

Olivier Rovellotti

\section{Presented at}

Biodiversity_Next 2019

\section{References}

- Chataigner J, Robert S, Poncet L, Vest F (2014) Règles sémantiques d'échange de données entre le SINP et le GBIF. Principes généraux et mappings. https://inpn.mnhn.fr/ docs/standard/sinp echangesinpdwc 2014.pdf

- GeoNature (2019) GeoNature, un outil open source développé par les parcs nationaux français. https://geonature.frl

- Jomier R, Poncet L, Robert S, Milon T, Archambeau A, Pamerlon S, Bourgoin T, Monicault LD, Barneix M, Fromage P, Carpy B, Callou C, Candelier S, Huguet A, Meunier D, WódkaGosse A, Grossiord F, Guichard B, Landrieu G, Lafage B (2018) Standard de données SINP Occurrences de taxons. 2.0. UMS 2006 "Patrimoine naturel", Paris, 100 pp.

- Legifrance (2016) LOI n 2016-1087 du 8 août 2016 pour la reconquête de la biodiversité, de la nature et des paysages. https://www.legifrance.gouv.fr/eli/loi/2016/8/8/2016-1087/jo/ texte

- Muséum national d'Histoire naturelle (2019) Presentation of the National Inventory of the Natural Heritage. https://inpn.mnhn.fr/accueil/presentation-inpn

- Natural Solutions (2019) Solution numérique pour gérer les données de terrain. https:// www.natural-solutions.eu/ecoreleve

- Wieczorek J, Bloom D, Guralnick R, Blum S, Döring M, Giovanni R, Robertson T, Vieglais D (2012) Darwin Core: An Evolving Community-Developed Biodiversity Data Standard. PLoS ONE 7 (1): 29715. URL: https://doi.org/10.1371/journal.pone.0029715

- Wilkinson M, Dumontier M, Aalbersberg IJ, Appleton G, Axton M, Baak A, Blomberg N, Boiten J, da Silva Santos LB, Bourne P, Bouwman J, Brookes A, Clark T, Crosas M, Dillo I, Dumon O, Edmunds S, Evelo C, Finkers R, Gonzalez-Beltran A, Gray AG, Groth P, Goble C, Grethe J, Heringa J, 't Hoen PC, Hooft R, Kuhn T, Kok R, Kok J, Lusher S, Martone M, Mons A, Packer A, Persson B, Rocca-Serra P, Roos M, van Schaik R, Sansone S, Schultes E, Sengstag T, Slater T, Strawn G, Swertz M, Thompson M, van der Lei J, van 
Mulligen E, Velterop J, Waagmeester A, Wittenburg P, Wolstencroft K, Zhao J, Mons B (2016) The FAIR Guiding Principles for scientific data management and stewardship.

Scientific Data 3 https://doi.org/10.1038/sdata.2016.18 